\title{
Swarm Patterns: Trends and Transformation Tools
}

\author{
Blesson Varghese and Gerard McKee \\ School of Systems Engineering, University of Reading, Whiteknights Campus \\ Reading, Berkshire, \\ United Kingdom
}

\section{Introduction}

The domain of multi-robot systems incorporates research which is inspired by nature. The aim of this research is to design man-made systems which incorporate principles observed in multi-agent natural systems. The mapping of these principles to man-made systems is referred to as biomimetics (Habib et al., 2007) and the area within multi-robot systems that explores this mapping is generally referred to as swarm robotic systems (Sahin \& Spears, 2005).

Research within swarm robotics includes self-organisation and an interesting aspect of self organisation is pattern formation (Camazine et al., 2003). The term pattern formation in literature is used in at least two different ways. Firstly, to define an area of study within multi robot systems that covers distinct aspects of patterns such as the establishment, maintenance and reconfiguration of patterns. Secondly, to report the natural phenomenon of flocking whereby loose or deformed geometric patterns emerge, and not necessarily strict geometric patterns (Arkin, 1998). In this chapter, the first usage of the term is adopted.

The research in this chapter is motivated by two observations based on an extensive review of literature based on pattern formation and swarm robotics. Firstly, it was noted that there are no mathematical models that exist for pattern formation in swarm robotic systems. Secondly, it was also noted that though pattern formation was a classic area of research, yet the challenges that have emerged in due course have not been addressed through a unifying model. Hence, it was necessary to address the need for a unifying mathematical model that can surmount the identified challenges in pattern formation.

The remainder of this chapter is organised as follows. The next section presents eight challenges identified in pattern formation. The second section sets the basic framework for mathematical modelling required to address the challenges. The fourth section presents a definition for transformation, four cases of transformation and tools for transformation. Simulation studies and a discussion are presented in the penultimate section. The last section concludes this chapter.

\section{Challenges of pattern formation}

With the progress of research in pattern formation a number of challenges have been identified by researchers. This section presents eight challenges which have been considered in exploring the need for a unifying mathematical model to address these challenges. The 
challenges were identified by reviewing extensive literature in swarm robotic systems, though only the most relevant of these are referenced here.

\subsection{Establishment of a pattern}

The problem of establishing patterns can be separated into two sub problems:

a. Identification of robots forming a pattern, which is based on the perceptual ability of the participating agents. The agents must recognize and establish communication with their peers with the goal of forming a single connected network (Poduri \& Sukhtame, 2007). The search for peer robots in an environment can be performed by random walks, but at the expense of battery power; hence the random walk approach is more appropriate for a closed environment with specified boundaries.

b. Positioning of the robots in the pattern requires a referencing mechanism such as the unit centre reference, leader reference, virtual reference and neighbour reference (Balch \& Arkin, 1998)(Michaud et al., 2002). In the Unit centre reference, each robot in a pattern computes a unit centre by averaging the $x$ and $y$ positions of other robots. In the leader, the formation position of a robot with respect to a lead robot position is determined. In the virtual reference mechanism, a virtual (or imaginary) point is referenced such that formation positions are based on this single point. In the neighbour reference mechanism, the formation position of a robot is determined with reference to one or more robots in close vicinity. Neighbour referencing can be based on a neighbour in the pattern that is the closest or farthest, or all detected neighbours.

\subsection{Maintenance of a pattern}

Once a pattern is established, the group of robots must move such that the pattern is maintained. Maintenance of a pattern can be considered as follows:

a. Stability of the pattern is challenged when an error is introduced into the pattern which is then propagated through the pattern resulting in the deformation of the pattern and possible failure in inter robot communication. String, mesh and leader to formation stability need to be studied in the context of patterns (Chen \& Wang, 2005)(Naffin et al., 2004). String stability deals with the effect of disturbance propagations in platoon formations and a stable formation requires the dampening of disturbance while it travels from any source. Mesh stability is used for error attenuation. Leader to formation stability deals with how the leader behaviour affects the interconnection errors.

b. Self-repairing ability is challenged when an unpredicted or untimely failure occurs. In such cases the unaffected robots in the pattern must be capable of coping with the loss. The group can continue motion towards its goal if a restoration of pattern is not necessary. If fixture and refurbishment of the pattern is required, reassigning pivotal roles and transforming patterns or repositioning robots is appropriate. The self repairing property of patterns has been demonstrated by (Cheng et al., 2005). In the event of failure of a group of agents in the pattern, the remaining agents adjust their positions such as to maintain uniform density.

\subsection{Obstacle avoidance}

Most research work on obstacle avoidance are focused on avoiding stationary obstacles. The complexity of the problem of obstacle avoidance increases when obstacles are dynamic in 
the environment. The problem of obstacle avoidance can be separated into obstacle identification, sharing obstacle information across the group and responding appropriately to the obstacle.

Obstacle identification is related to the perceiving ability of the robotic agents in the pattern. For example, obstacles can be identified through vision mechanisms. Sharing obstacle information across the group can be achieved by broadcasting to all agents (global communication), or to a subset of a group, or the agents individually sense information of obstacles and respond locally. The solutions to these two problems provide the base for the appropriate response to the obstacle. Strategies employed for avoiding obstacles in patterns include potential field (Wang et al., 2006), dynamic window (Fox et al., 1997)(Seder \& Petrovic, 2007)(Ogren \& Leonard, 2003) and flow field method (Shao et al., 2006).

\subsection{Collision avoidance}

The chance of robots colliding against each other is yet another challenge in robot formations. Some researchers use the term collision avoidance synonymous with obstacle avoidance (Cai et al., 2007a)(Cai et al., 2007b). However, we treat the avoidance of inter robot collisions as collision avoidance.

Collisions are avoided by maintaining strict buffer distances and consistent communication between robots (Koh \& Zhou, 2007). Maintaining strict buffer distances can challenge the flexibility of the pattern. Hence there is a trade off between flexibility and buffered distance for collision avoidance. Strict collision avoidance rules might prevent the establishment of a desired pattern.

\subsection{Transformation of patterns}

The transformation of patterns is synonymous with reconfiguration of patterns which is necessary when a swarm of robots need to respond to obstacles in the path of its motion (Chen \& Wang, 2007b). Reconfiguration can be achieved by repositioning all or a few agents in the pattern and can lead to the deformation of a pattern or a change of shape of the pattern. When many robotic agents within a pattern attempt to reposition, chances of inter agent collisions increase. Hence collision avoidance discussed in the previous section is relevant while repositioning and may require path planning of individual robots.

However, we treat the transformation of patterns distinct from mere repositioning, since transformations are more geometry oriented. Repositioning of agents may be feasible only on flexible patterns while transformations can be achieved on both rigid and flexible patterns. The transformation of patterns, of primary focus in this chapter, is considered later in Section 4.

\subsection{Role assignment}

This challenge involves designating a role or assigning a job to a robot or a group of robots (Chaimowicz et al., 2002)(Chen \& Wang, 2007a). Consider the example of a group of robots patrolling a secure area. It is possible that there exists a single assigned leader for the group. All the followers of the leader in the pattern perform a coordinated task and achieve the goal. But consider a team of robots in a soccer match. Each robot has its own specific role. At any instant, a robot would assume the role of a goal keeper, while some act as defenders or strikers. Hence, the role assigning module of a framework is of important consideration. 
Group of robots in a pattern assigned roles should be capable to swap roles and accommodate heterogeneous robots in the pattern. Robust frameworks would require strategies to reassign roles at the event of agent failure or even while avoiding obstacles.

\subsection{Scalability of patterns}

Most pattern formation research is based on a finite number of robots and has proven to be not scalable. It is also interesting to note that the issue of scalability has been of little interest in most research work. It is practically impossible to study scalable formations on real world robot systems because of the cost factor. However, there is a need to derive models that facilitate scalability studies and are scalable.

Simulations would be an appropriate method to study scalability of patterns for which various numerical techniques and simulation environments are available. Robotic simulations necessarily need not be only performed on robotic development environments but may also employ physics, chemical or biological simulators. A particle physics based swarm simulation study is reported in (Varghese \& McKee, 2008b).

\subsection{Coordinating multiple patterns}

Coordinating robots in a single group for a cooperative task has been of interest in swarm robotic research. It would also be interesting to consider multiple groups forming independent patterns for cooperative tasks. This includes coordination amongst groups of robots to merge and split to different patterns. Hence, the need for multiple role assignment strategies arises. Sharing of agents between groups at the event of agent failures could be a further consideration towards developing a robust strategy for multiple pattern coordination. The coordination of multiple teams (Hsu \& Liu, 2004) and task assignment of multiple agents, pattern splitting and merging while accomplishing a task have been recently reported (Michael et al., 2008).

Researchers have attempted to address the eight challenges presented above with significant research contributions towards classic challenges such as obstacle and collision avoidance. However, it is noted that attempts towards developing a model that addresses most of the above challenges has been minimal. Hence, there exists a need to develop a mathematical model towards this end, such that the challenges presented above can be addressed.

\section{A mathematical model for swarm patterns}

This section proposes a mathematical model for swarm pattern formation based on the foundations of the Complex Plane and is shown in figure 1. The De Moivre's formula to obtain roots of an equation is used to represent the model. If $z=x+$ iy (Kreyszig, 2006) and is represented in the polar form as $\mathrm{z}=\mathrm{r}(\cos \theta+i \sin \theta)$ and $\mathrm{r}$ is called the absolute value or the modulus of $\mathrm{z}$, then $\mathrm{z}^{n}=\mathrm{r}^{n}(\cos n \theta+i \sin n \theta)$ for $\mathrm{n}=0,1,2, \ldots$ The $\mathrm{n}^{\text {th }}$ root of $\mathrm{z}$ is obtained by $\sqrt[n]{z}=\sqrt[n]{r}\left(\cos \left[\frac{\theta+2 k \pi}{n}\right]+i \sin \left[\frac{\theta+2 k \pi}{n}\right]\right)$ where $\mathrm{k}=0,1, \ldots,(\mathrm{n}-1)$. The roots of the equation lie on a circle of radius $\sqrt[n]{r}$ with centre at the origin and constitute the vertices of a regular polygon of $\mathrm{n}$ sides. The result of joining the $\mathrm{n}$ roots is an $\mathrm{n}$-sided polygon. The 
polygon is circumscribed by a circle otherwise referred as the circumcircle of the polygon. Mapping these results on to the area of multi-agent pattern formations, it is assumed that the robotic agents are positioned on the vertices of the polygon. Hence the robots form a closed polygonal pattern and the system is mobile with appropriate communication and coordination mechanism.
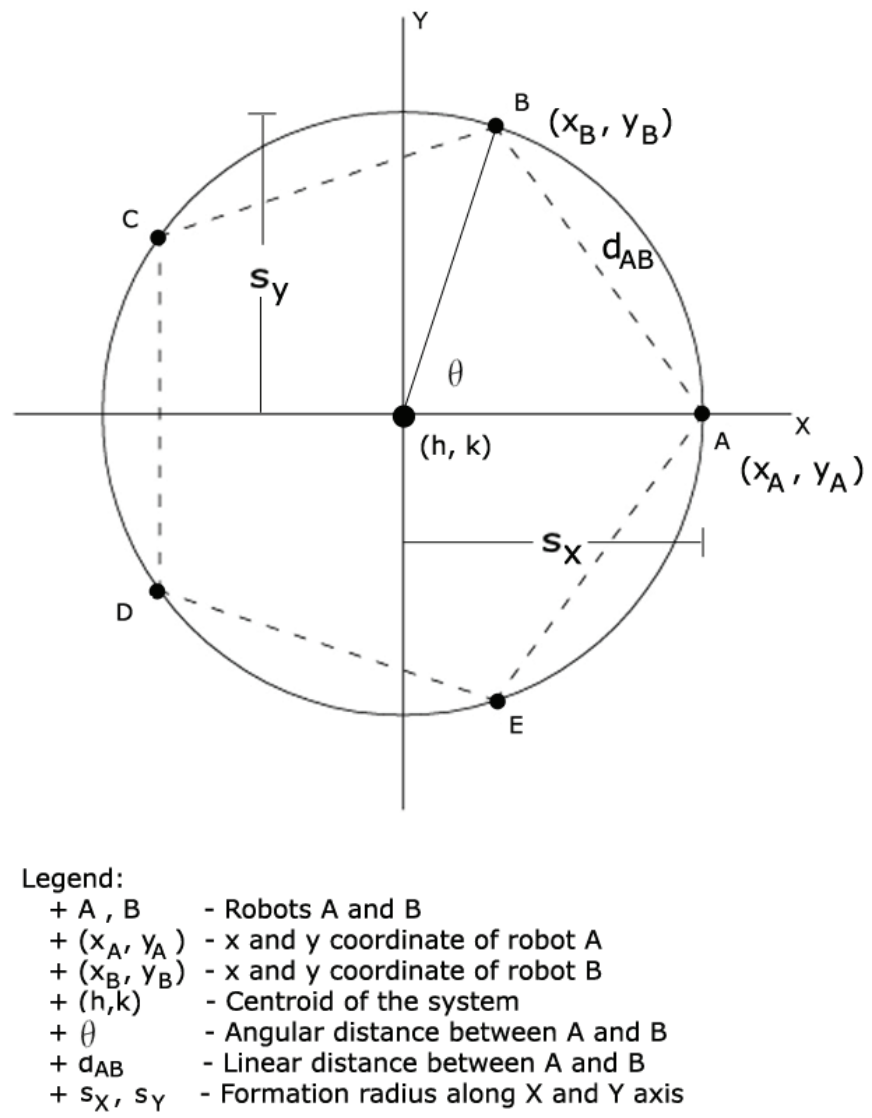

Fig. 1. Mathematical Model for Swarm Pattern Formation

Before proceeding further it is necessary to define a few terms such as microscopic and macroscopic primitives to articulate the mathematical model proposed in this section. The term primitive in this paper refers to an element used as a building block to define aspects of the model.

Microscopic primitives are specific to robots constituting the swarm and define the individual behaviours of swarm robotic agents. Microscopic primitives are employed in the research reported by (Chen et al., 2005)(Balch \& Hybinette, 2000). It is also noted that behaviour based pattern formation approaches tend to be microscopic in nature and such models may not be scalable. 
On the other hand macroscopic primitives of a group of robots are properties that affect the group behaviour of the system. They are abstract properties of a pattern, which when modified facilitates a change in the pattern. The control of a swarm of robot by varying abstract properties, namely variance and centroid is reported by Belta and Kumar (Belta \& Kumar, 2004).

There are atleast four benefits of using macroscopic primitives. Firstly, implicit coordination, which refers to the coordination of a pattern comprising of mobile robots, need not be specified externally. Coordination is achieved as a result of varying the macroscopic primitives. Secondly, Group behaviour definition, which refers to the collective behaviour of the group, is possible by controlling the macroscopic primitives. The individual behaviour of the units is affected by the variation in the macroscopic primitive. Thirdly, Adaptability, which refers to the ability of the group to adjust to change of internal or external circumstances, can be affected by macroscopic primitives. Fourthly, Stability, which refers to the factor by which the robot group maintains a pattern, can be controlled by using macroscopic primitives to dampen the propagation of errors.

The mathematical model is realized by considering macroscopic primitives. The macroscopic primitives are separated into primary and secondary primitives. Primary macroscopic primitives are basic or fundamental elements. They are considered as input variables to the model and are irreducible to simpler parameters or expressions and therefore termed as independent primitives. Secondary macroscopic primitives are derived from other primitives of the mathematical model. Hence, these primitives are termed as dependent primitives.

The primary macroscopic primitives of the model proposed in this paper are the total number of robots, angular separation, formation radius and elongation. The total number of robots in a polygonal pattern, given by $n$, equates to the number of vertices of a polygon or the roots of the complex equation. Angular separation is an important factor that determines the coordinates for positioning robots in a polygonal pattern. Angular separation, denoted by $\theta$, is a measure of the angular spacing between adjacent robots of a pattern. Formation radius, denoted by $r$, is the radius of the circumscribing circle of the polygonal pattern. This primitive determines the area occupied by the pattern. Elongation ratio of a pattern, denoted by $e$, is a ratio of magnitudes of the major and minor axis of the pattern and quantifies the shape transforming behaviour of a pattern. The symmetry of a pattern can also be described by the elongation ratio.

The secondary macroscopic primitives are linear distance and shaping radii. The distance between adjacent robots in the polygon is a constant if the polygon is regular. To compute the distance between robots, the coordinate positions of the robot need to be known. The centroid of the pattern, $(h, k)$, is used to compute the coordinates of robots. Further, the Euclidean distance between adjacent robots $A$ and $B$ is given by $d_{A B}=\sqrt{\left(x_{B}-x_{A}\right)^{2}+\left(y_{B}-y_{A}\right)^{2}}$. Hence, linear distance is dependent on the position coordinates of robots.

The shaping radii along the $x$ and $y$ axis, $s_{x}$ and $s_{y}$ respectively determine the measure of deflation or inflation of a pattern laterally and longitudinally. The magnitudes of elongation and formation radius are useful to determine the shaping radii of a pattern and are given by $s_{x}=r e$ and $s_{y}=\frac{r}{e}$. The equations that define the shaping radii are also given by 
$x_{B}=h+s_{x} \cos \theta$ and $y_{B}=k+s_{y} \sin \theta$. Hence, orientation radii are dependent on formation radius and elongation.

\section{Pattern transformation}

Having established a model for pattern formation in swarm systems it is also necessary to study and further develop the model to achieve transformation of patterns. This section defines the challenge of transformation and presents four cases of transformation.

\subsection{Definition}

Consider a rigid pattern $P$ with geometric relationships represented as $G_{P}$ which is macroscopic in nature such that the relationship $G_{P}$ can be manipulated by varying some macroscopic parameter that relates to the pattern $P$. The pattern $P$ comprises $N$ robots such that their positions are given by $p_{i}\left(x_{i}, y_{i}\right)$ where $p_{i} \in R^{2}$ and $i=1,2, \ldots, N$. Pattern $P$ transforms into the pattern $Q$ with geometric constraints or relationships represented as $G_{Q}$ which is macroscopic in nature such that the relationship $G_{Q}$ can be manipulated by varying some macroscopic parameter that relates to the pattern $Q$. The pattern $Q$ also comprises $N$ robots such that the position of the robotic agents is given by $q_{i}\left(x_{i}, y_{i}\right)$ where $q_{i} \in R^{2}$ and $i=1$, $2, \ldots, N$.

The function which enables the transformation of the pattern $P$ to $Q$ is given by $f(P)=Q$. In other words, $f\left(p_{1}\left(x_{1}, y_{1}\right), p_{2}\left(x_{2}, y_{2}\right), \ldots, p_{N}\left(x_{N}, y_{N}\right)\right)=q_{1}\left(x_{1}, y_{1}\right), q_{2}\left(x_{2}, y_{2}\right), \ldots, q_{N}\left(x_{N}, y_{N}\right)$. The application of an inverse transformation function on the transformed pattern $Q$ yields the pattern $P$, given by $f^{-1}(Q)=P$. The transformation on the pattern also results in a transformation of the geometrical relationships from to between the participating agents in the pattern.

\subsection{Transformation cases}

Four cases of transformation based on the above definition are derived by imposing restrictions on the geometrical constraints.

\subsubsection{Case 1}

$G_{Q}=G_{P}$ after a transformation that involves repositioning all agents. This case is relevant when robotic agents in the pattern have repositioned, yet the geometrical pattern has not changed. Such a transformation is termed as Elementary transformation in this paper. This term also refers to those transformations very basic in nature. For instance, a swarm could be rotated with respect to its centroid or translated such that all robotic agents have repositioned themselves. Though the orientation of the pattern has changed, the configuration of the pattern remains unaltered. Mathematically, the case of elementary transformation would be such that $G_{Q}=G_{P}$ and $\forall i: p_{i}\left(x_{i}, y_{i}\right) \neq q_{i}\left(x_{i}, y_{i}\right)$.

\subsubsection{Case 2}

$G_{Q}=G_{P}$ after a transformation without repositioning all agents. This case considers the rotation or translation of the swarm with respect to a few robotic agent whose position remains fixed. This case is also classified under elementary transformation, yet repositioning 
of all agents has not occurred. Mathematically, this case of elementary transformation would be such that $G_{Q}=G_{P}$ and $\exists i: p_{i}\left(x_{i}, y_{i}\right)=q_{i}\left(x_{i}, y_{i}\right)$.

\subsubsection{Case 3}

$G_{Q} \neq G_{P}$ after a transformation that involves repositioning all agents. This relates to the case when the geometrical constraints of the pattern have changed and a new pattern has emerged. It is termed a geometric transformation. This concept is relevant when robotic agents in the pattern reposition to result in a geometry change. For instance, the shape of a swarm could be geometrically transformed from a polygon to a line. It is interesting to note that the scaling of a pattern would result in a geometric transformation, since the geometrical constraints are dissimilar in both cases. Mathematically, the case of geometrical transformation would be such that $G_{Q} \neq G_{P}$ and $\forall i: p_{i}\left(x_{i}, y_{i}\right) \neq q_{i}\left(x_{i}, y_{i}\right)$.

\subsubsection{Case 4}

$G_{Q} \neq G_{P}$ after transformation without repositioning all agents. This case considers the geometric transformation such that the position of one or more than one robotic agent remains fixed. It is classified under geometric transformation, yet repositioning of all agents has not occurred. Mathematically, the case of geometrical transformation would be such that $G_{Q} \neq G_{P}$ and $\exists i: p_{i}\left(x_{i}, y_{i}\right)=q_{i}\left(x_{i}, y_{i}\right)$.

Cases 1 and 2 relate to elementary transformation of the pattern. In these cases, the pattern remains rigid in nature, since the geometric constraint or relationship persists even after elementary transformation. Cases 3 and 4 deal with geometric transformation and introduce flexibility into rigid patterns.

\subsection{Tools for pattern transformation}

This section considers two tools for pattern transformation, namely a macroscopic transformation tool and a mathematical transformation tool. Cases 1, 3 and 4 of transformation are considered in the both the transformation tools. To achieve geometrical transformation, a series of operations are performed in both methods. Case 2 of transformation will be reported elsewhere.

\subsubsection{Tool 1: macroscopic transformation}

The first transformation tool proposed in this section which is inclusive of both elementary and geometric transformations considers cases 1, 3 and 4 of transformation and are applied on the swarm model. This tool varies a macroscopic parameter, namely the formation radius (along $\mathrm{x}$ and $\mathrm{y}$ axis) of the swarm model to facilitate transformation. A sequence of operations is performed on the swarm model to obtain a transformed pattern and is shown in figure 2.

The set of operations are:

a. Rotation: The initial step of rotation of the model is performed to achieve collision avoidance during the next step. Apredefined angle offset is used to rotate the swarm. Though the robots are repositioned, the operation results in the same polygonal pattern with a different orientation from the former. Here, the concept of elementary transformation is introduced. Though all robots were repositioned in this operation, a 
geometric transformation is not evident since the shape of the pattern is retained. Though a geometric transformation is not evident, yet an elementary transformation of case 1 is achieved in this step.

b. Macroscopic Parameter Operation: Following a rotation operation, the macroscopic parameter is set to be modified. Deflating the model along the y-axis would result in a deformed polygonal pattern. The deflation of the model is performed by decrementing the magnitude of the formation radius along the y-axis. When deflation has reached its maximum value, the robotic agents have aligned themselves entirely along the $x$-axis. Maximum deflation is achieved when the formation radius value along any axis vanishes. An inflation operation along the other perpendicular axis simultaneously while deflating would result in a pattern with larger inter-linear distance between the agents (a measure for avoiding collisions). This variation is possible due to the notion of flexibility in rigid patterns.

c. Further Rotation: This step is performed to achieve equidistance between the participating agents. Though the pattern has transformed its shape by this step, the participating agents are still governed by the rules of the swarm model. A corrective rotation measure would ensure that the agents are loosely equidistant.

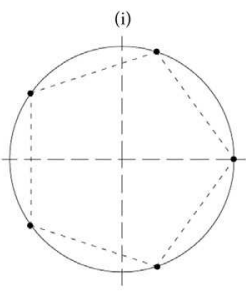

(v)

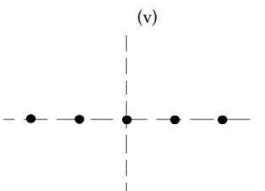

(ii)

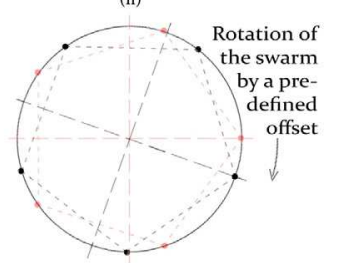

Legend:

+ (i) Original Swarm Model

+ (ii) Rotated Swarm Model (Original swarm model seen in background)

+ (iii) Deflated Swarm Model (by reducing the formation radius along y - axis)

+ (iv) Transformed Swarm Model

+ (v) Transformed Swarm Model with equidistant spacing between robots

Fig. 2. Sequence of operations for a circle-to-line transformation using the Macroscopic Transformation Tool

\subsubsection{Tool 2: mathematical transformation}

The method proposed in this section considers case 3, which is achieved by using a mathematical transformation tool. Many mathematical tools are available for transformations such as stretching, rotating, reflecting and translating. The linear fractional transformation is one such readily available mapping function that maps a set of points from one plane to another. The transformation is given by $\frac{a z+b}{c z+d}$, where $z, a, b, c$ and $d$ are complex numbers satisfying $a d-b c \neq 0$. The linear fractional transformation is also known as a Moebius transformation (Kreyszig, 2006).

The transformation functions are applied onto the swarm pattern which is polygonal in shape. Since the vertices of the polygonal pattern lie on the circle circumscribing the pattern, a circleto-line and a line-to-circle are the two transformation tools employed on the complex 
plane. However, the transformation function cannot be applied directly to the swarm pattern since the mathematical function is applicable on the local frame of reference and the swarm pattern in the proposed model is defined on the global frame of reference. Hence, a sequence of operations are performed on the swarm pattern to achieve transformation and is shown in figure 3 . The set of operations are:

a. Transformation from global to local frame of reference: The frame of reference of the swarm is temporarily transformed from the global to a local frame. The local frame of reference considered is such that the circumscribing circle is divided into four equal quadrants. Hence the centroid of the pattern lies on the origin position of the local frame.

b. Discrete Transformation: This step applies the mathematical transformation function on the swarm model. The transformation of a circle to a line is obtained from $w=i \frac{1-z}{1+z}$. Applying the equation on the Euclidean plane, the mapping function is deduced as $\left(\frac{2 y}{1+x^{2}+y^{2}}, \frac{1-x^{2}-y^{2}}{1+x^{2}+y^{2}}\right)$. The transformation from a line to a circle is applied by considering a special case of the Moebius transformation. The transformation $w=\frac{1}{z}$ maps every straight line or circle onto a circle or straight line. It is also known as the inversion in the unit circle or reciprocal transformation. Applying the equation on the Euclidean plane, the mapping function is otherwise written as $\left(\frac{x}{x^{2}+y^{2}}, \frac{y}{x^{2}+y^{2}}\right)$. The destination coordinates obtained by the mathematical functions are the coordinates to which individual robot agents need to reposition while the pattern transforms. However, it is evident that these transformation functions are discrete in nature yielding only one set of destination coordinate rather than sub-goals or intermediate destination coordinates.

c. Transformation from local to global frame of reference with magnification: The destination coordinates are obtained on the local frame of reference. Hence, the local frame needs to be shifted to the global frame of reference. Since the mathematical functions considered in operation (b) are reducing functions (destination coordinates reduce the span of the pattern), a magnification ratio is used in the local frame to achieve gain in the destination coordinates.

d. Path planning by discretization: Since the achieved destination coordinate set is discrete, the major challenge in repositioning agents is to plan their path to the destination coordinates. In this paper, the technique adopted to reposition robots is along straight line trajectories without collisions. The straight line path between the agent and its estimated destination is discretized. Figure 4 illustrates the straight line discretization process. The domain values of the path are sliced to extrapolate the range values. This relates to the underlying principle of Discrete Event Simulations (DEVS). The potential of DEVS in path planning for robots has been reported by (Arikan et al., 2001).

\section{Simulation studies}

Simulation studies were pursued to validate and visualize the mathematical model and tools employed for pattern transformation. The feasibility of the proposed approach was 


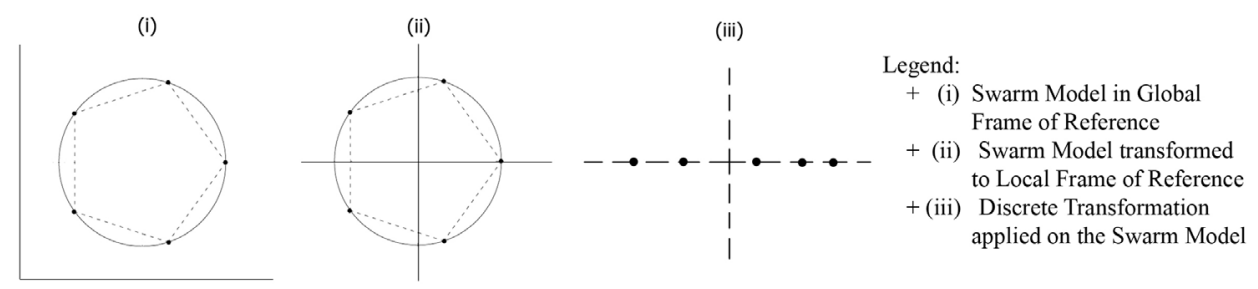

Fig. 3. Sequence of operations for a circle-to-line transformation using the Mathematical Transformation Tool
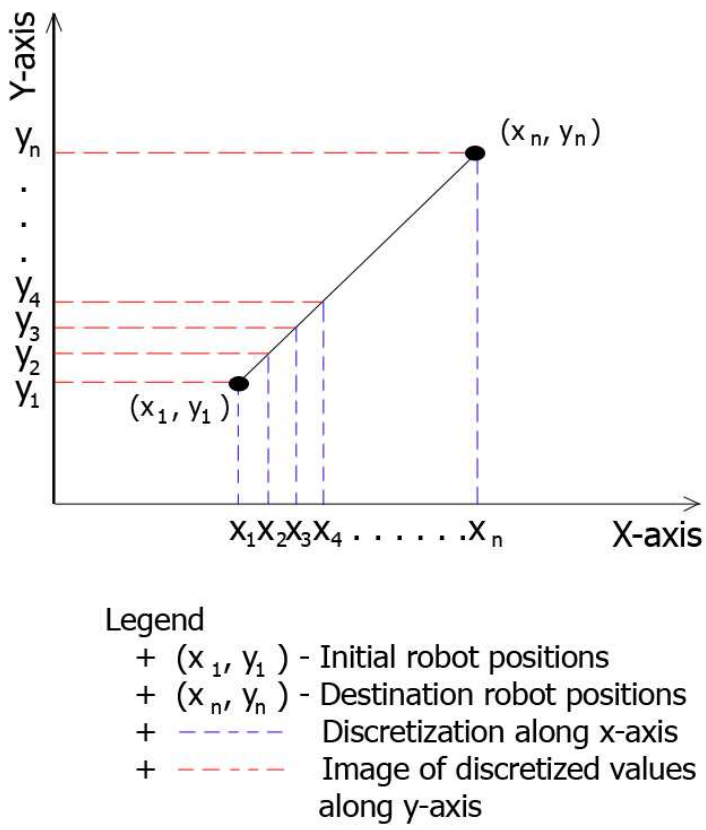

Fig. 4. Discretization of a straight line

validated on the Processing (Processing website, 2010) and Traer Physics (Traer Physics website, 2010) environment. Processing is an open source programming language and environment enabling visualizations for learning and prototyping, where as Traer Physics is a particle physics simulation engine for Processing.

The Traer physics library has provisions for modeling a particle system, particles, springs and attractive or repulsive forces (Traer Physics website, 2010). The particle system enables to prototype particles and forces. Particles represent objects which are stationary or dynamic in an environment and can be modelled using four properties, namely mass, position, velocity and age. Springs on the other hand can connect two particles and prevent collisions. Springs are characterized by three properties, namely rest length, strength and damping. Attractions or repulsions pull particles together or apart and have two properties, namely 
strength and minimum distance. The simulations reported in this paper employ the particle system, particles and attractive or repulsive forces.

\subsection{Experimental setup}

To establish a pattern, the particles of the pattern are modelled in an open environment such that they are acted upon by forces, namely macro and micro level forces of attraction and repulsion. The macro level forces include repulsive forces, which act on the centroid of the swarm and maintains the stability of the pattern. The forces of repulsion are generated from obstacles (modeled as forces) in the environment. All robotic agents align themselves around the centroid with respect to the forces forming a virtual structure polygonal pattern. Obstacles in the path of the pattern are detected by the computation of the net force acting on the group of robots. Beyond a maximum threshold of force, the pattern reacts appropriately by transforming its shape to avoid obstacles. The pattern regains its polygonal shape when the net force acting on the centroid decreases below a minimum threshold value, such as when the pattern has escaped from obstacles. The inter-agent bonding force and the forces of interaction with the centroid contributed to the micro level forces. The pattern generates a propulsive force to trace paths against repulsive forces.

The experimental setup comprised a tunnel through which the swarm had to displace. The walls of the tunnel generated repulsive forces and acted as the obstacle. The swarm initiated its motion from the left of the tunnel and aimed to reach a goal beyond the tunnel on the right side. While attempting to pass through the tunnel, the swarm transformed its shape to avoid obstacles and avoided collisions between agents. Both transformation tools discussed in the previous section were implemented.

First of all, the macroscopic transformation tool which consists of a sequence of three operations was implemented. Firstly, the swarm model was rotated to avoid collisions while deflating. Table 1 illustrates the different rotation angles that were applied on the swarm. Higher value angles resulted in collisions for most patterns. Angles less than 15 degrees proved effective for collision avoidance.

Secondly, the macroscopic parameters were varied. This variation resulted in deflation or inflation of the pattern (along $\mathrm{x}$ or $\mathrm{y}$ axis). Thirdly, a corrective rotation was applied to avoid agents from colliding against each other. Hence by transformation of the pattern, the swarm successfully displaced itself through the obstacle path. Figure 5 is a snapshot of the

\begin{tabular}{c|c|c|c|c}
\hline \multirow{2}{*}{$\begin{array}{c}\text { Angle Offset } \\
\text { No. of Robots }\end{array}$} & \multicolumn{1}{|c|}{$35^{\circ}$} & $40^{\circ}$ & $60^{\circ}$ \\
\cline { 1 - 4 } 3 & - & - & - & 1 \\
4 & - & - & 2 & - \\
5 & - & 1 & - & - \\
6 & - & 3 & - & 3 \\
\hline
\end{tabular}

Table 1. Rotation Values \& Estimated Collisions 
(i)
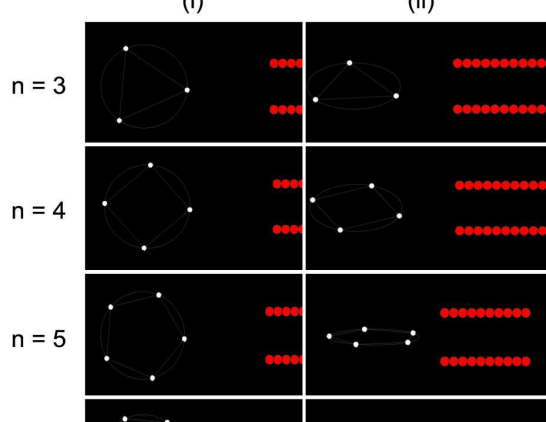

$n=6$

$\mathrm{n}=20$

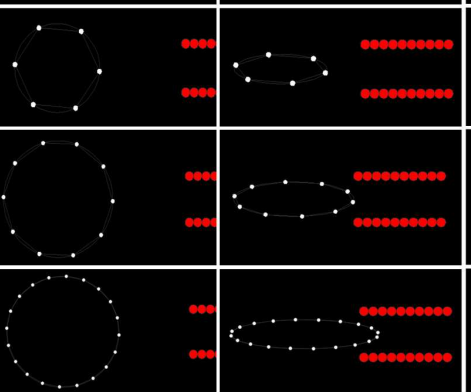

(iii)

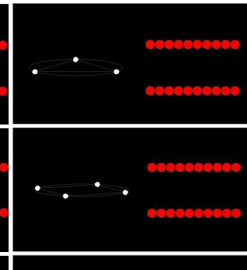

(iv)

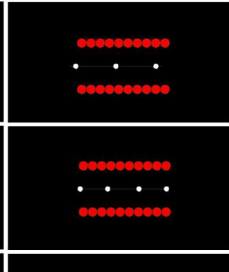

(v)

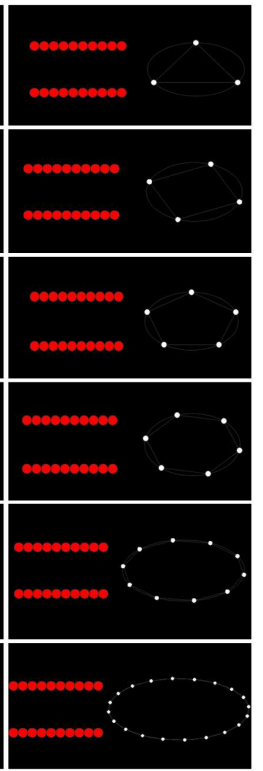

Fig. 5. Simulation results for transformation using Macroscopic Transformation tool. (i) Rotated swarm model for various number of robots, (ii) Deflation of the model along the yaxis (For $\mathrm{n}=10$ and 20, inflation along $\mathrm{x}$-axis performed), (iii) Transformed pattern without corrective rotation measure, (iv) Transformed pattern after corrective rotation measure is applied (Except for $\mathrm{n}=3$ and 4 , since equidistance is more or less achieved), (v) Inverse transformation by inflation back to original pattern.

simulation studies for $\mathrm{n}=3$ to 6,10 and 20 robots transforming when the first transformation tool is employed.

It is observed that a geometric transformation is obtained by performing a sequence of operations which consists of an elementary transformation. A regular pattern transforms to an irregular polygonal pattern while reconfiguring.

Then, the mathematical transformation tool which consists of a sequence of four steps was implemented. Firstly, the swarm pattern was transformed from the global to a local frame such that the centroid of the pattern lies on the origin of local frame of reference. Hence, the pattern is equally spanned over the four quadrants in the local frame of reference, which was necessary for proper implementation of the transformation functions.

Secondly, the discrete transformation function was applied on the microscopic property, namely the position coordinates of the individual robots in the pattern. The transformation from a circle to line was employed in order for the pattern to pass through the tunnel in the environment. Beyond the obstacles, the transformation from a line to circle was employed. Both transformation operations yield a set of discrete destination coordinates for each robot. Thirdly, transformation from the local to global frame of reference was performed. The destination coordinates obtained in the local frame of reference were such that the pattern radius is reduced. Hence a magnification of the coordinates in the local frame was performed and further mapped on to the global frame of reference. 


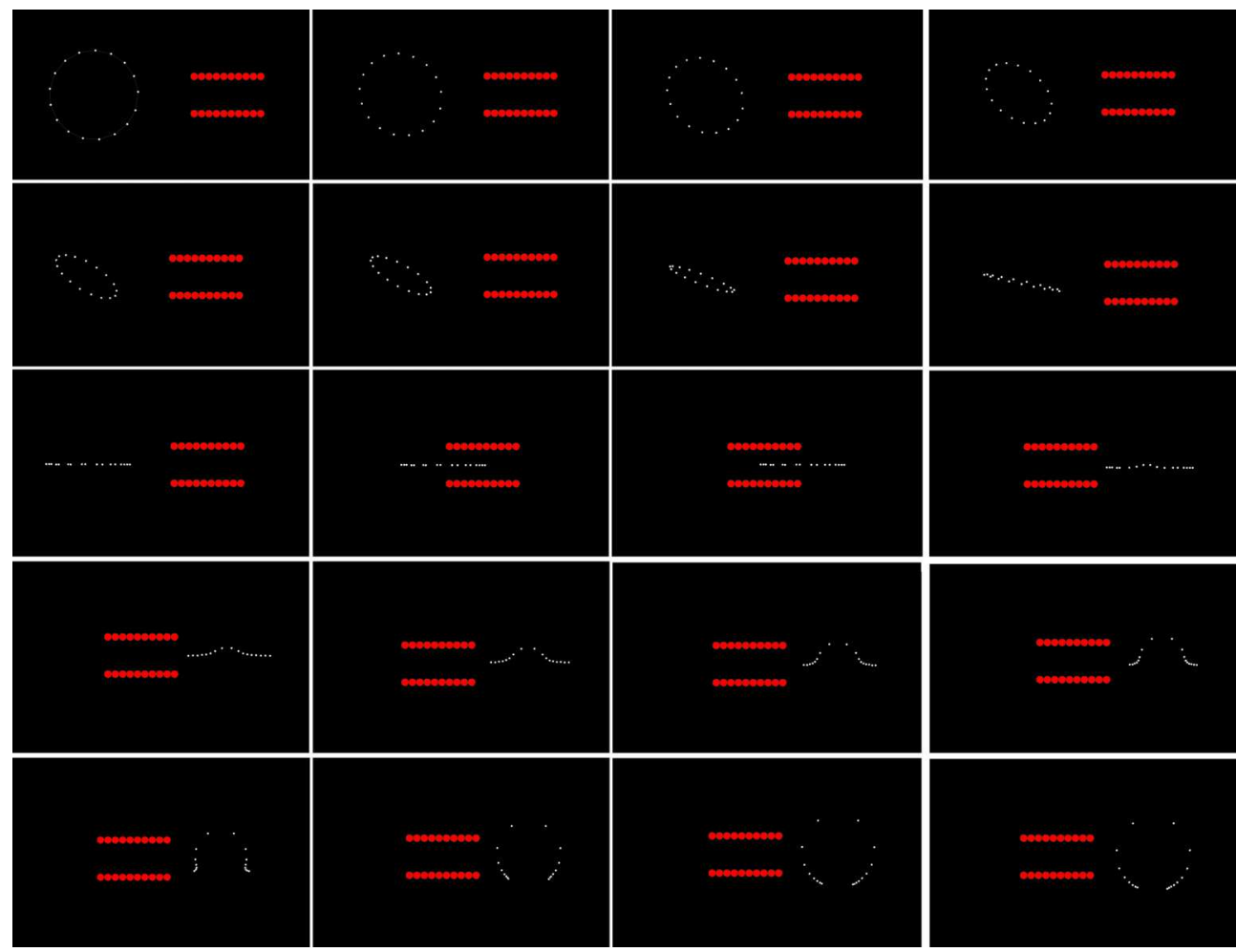

Fig. 6. Simulation studies for transformation using the Mathematical Transformation tool. Firstly, a circle to a line transformation (First three rows excluding the fourth sequence in the third row). Secondly, a line to circle transformation (Fourth sequence of the third row and last two rows).

Fourthly, path planning by discretization was executed. This step is essential to determine the sub goals or intermediate position coordinates. Repositioning the robots to sub-goals or intermediate coordinates is a computationally expensive process. Straight line trajectories from agents to calculated destination coordinates without collisions were considered in the work reported in paper. Figure 6 is a snapshot of the simulation studies for 17 robots that transform shape when the second transformation tool is employed.

It is observed that the circle to line transformation yielded a pattern in which robotic agents were loosely equidistant. The line to circle transformation employing the reciprocal transformation yielded a polygon irregular in nature. This was due to the nature of the reciprocal transformation which was anticipated.

It was observed that in both methods, the swarm successfully displaced itself through the obstacle path by transforming shape. The transformed patterns were loosely equidistant. Collision avoidance between repositioning agents is not implicitly guaranteed. Hence, atleast one operation in both methods ensured collision avoidance. The geometrical transformation of a circle to a line in both cases was achieved by transforming a regular polygonal pattern to an irregular pattern by repositioning agents. The observations are 
consistent with the theoretical studies presented in the previous section and according to the authors expectation.

\subsection{Discussion}

The transformation methods presented in this chapter are feasible tools for transforming patterns. However, it is noted that the mathematical tool employing Moebius transformation is not strictly macroscopic in nature; the microscopic properties of the swarm units are taken into consideration. For example, path planning of individual robots is necessary to reposition the robots. The method is not advantageous for small number of robots in the pattern. Moreover the mathematical transformation tool is a discrete transformation method. Hence, discretizing and quantizing the path for repositioning robots are required which are both computationally expensive operations unsupported and unwarranted on minimal processing swarm units. Therefore global planning is required thereby increasing wireless communication overheads. A high bandwidth for communication and synchronized and consistent communication with a centralized unit are challenges in realizing the mathematical tool in real time.

On the other hand, the macroscopic tool considers the group behaviour of the swarm system. Hence, individual robots need not be addressed, thereby eliminating microscopic parameter operations. For example, transformation using the macroscopic tool is obtained by a sequence of operations performed on the entire swarm pattern rather than considering individual robot path planning. The macroscopic tool is also observed to be consistent in the time taken for transformation, and is also continuous thereby reducing computations for individual robot planning. This tool would hence offer better synchronization between the swarm units since local planning is sufficient. Hence wireless communication overheads are relatively less compared to the mathematical tool.

By implementing an approach that could incorporate the macroscopic tool in a real time robot system, planning overheads for individual robots could be minimized. However, a mathematical transformation function is advantageous since it belongs to an analytical class of tools and mathematical analysis is possible. Therefore, this approach will also be explored in future work.

\section{Conclusion}

In summary, a review of the literature has revealed that there are at least eight challenges in pattern formation that need to be addressed. Further, a single mathematical model that aims to address these challenges are unavailable since research in this direction has not been a key focus. Hence, the work reported in this chapter is motivated by the need for developing a mathematical model that attempts to address the challenges in pattern formation. Particularly, transformation of patterns has been considered in this chapter. A mathematical model for pattern formation based on the complex plane is proposed. A definition for transformation, four cases of transformation and two tools for transforming patterns in the mathematical model are proposed. Simulation studies employing a particle physics simulation engine has incorporated the mathematical model. These studies have proven that most challenges in pattern formation can be addressed using the mathematical model, thereby validating the feasibility of the model. The challenge of role assignment, though not 
considered in this chapter, is reported elsewhere (Varghese \& McKee, 2008a). Scalability studies are not presented in this chapter. Coordinating multiple patterns using the mathematical model need to be addressed in the future.

\section{References}

Arikan, O.; Chenney, S. \& Forsyth, D. A. (2001). Efficient Multi-Agent Path Planning, Proceedings of the Eurographic Workshop on Computer Animation and Simulation, Manchester, UK, pp. 151- 162.

Arkin R. C. (1998). Behavior-Based Robotics. The MIT Press.

Balch, T. \& Arkin, R. C. (1998). Behavior-based formation control for multi robot teams, IEEE Transactions on Robotics and Automation, pp. 926-939.

Balch, T. \& Hybinette, M. (2000). Social potentials for scalable multi-robot formations, Proceedings of the IEEE International Conference on Robotics and Automation, pp. 73-80.

Belta, C. \& Kumar, V. (2004). Abstraction and Control for Groups of Robots, IEEE Transactions on Robotics, pp. 865-875.

Cai, C.; Yang, C.; Zhu, Q. \& Liang, Y. (2007). Collision avoidance in multi-robot systems, Proceedings of the IEEE International Conference on Mechatronics and Automation, pp. 2795-2800.

Cai, C.; Yang, C.; Zhu, Q. \& Liang, Y. (2007). A Fuzzy-based collision avoidance approach for multi-robot systems, Proceedings of the International Conference on Robotics and Biomimetics, pp. 1012-1017.

Camazine S.; Deneubourg J. - L.; Franks N. R.; Sneyd J.; Theraulaz G. \& Bonabeau E. (2003). Self-Organization in Biological Systems, Princeton University Press.

Chaimowicz, L.; Campos, M. F. M. \& Kumar, V. (2002). Dynamic Role assignment for cooperative robots, IEEE International Conference on Robotics and Automation, pp. 293-298.

Chen, Y. Q. \& Wang, Z. (2005). Formation control: a review and a new consideration, Proceedings of the IEEE International Conference on Intelligent Robots and Systems, pp. 3181-3186.

Chen, Y. - C. \& Wang, Y. - T. (2007). Dynamic role assignment algorithm for robot formation control, IEEE International Conference on Advanced Intelligent Mechatronics, pp. 1-6.

Chen, Y. - C. \& Wang, Y. - T. (2007). Obstacle avoidance and role assignment algorithms for robot formation control, Proceedings of the IEEE International Conference on Intelligent Robots and Systems, pp. $4201-4206$.

Chen, P.; Song, Z.; Wang, Z. \& Chen, Y. Q. (2005). Pattern formation experiments in mobile actuator and sensor, Proceedings of the IEEE International Conference on Intelligent Robots and Systems, pp. 735-740.

Cheng, J.; Cheng, W. \& Nagpal, R. (2005). Robust and Self-Repairing Formation Control for Swarms of Mobile Agents, Proceedings of the 20th AAAI National Conference on Artificial Intelligence, pp. 59-64.

Fox, D.; Burgard, W. \& Thrun, S. (1997). The dynamic window approach to collision avoidance, IEEE Robotics and Automation Magazine, pp. 23-33. 
Habib, M. K.; Watanabe, K. \& Izumi, K. (2007). Biomimetics Robots from Bio-inspiration to Implementation, Proceedings of the 33rd Annual Conference of the IEEE Industrial Electronics Society

Hsu, H. C. - H.\&Liu, A. (2004). Multiple teams for mobile robot formation control, Proceedings of the IEEE International Symposium on Intelligent Control, pp. $168-173$.

Koh,W. L. \& Zhou, S. (2007). An Extensible collision avoidance model for realistic selfdriven autonomous agents, Proceedings of the IEEE International Symposium on Distributed Simulation and Real- time applications, pp. 7-14.

Kreyszig, E. (2006). Advanced Engineering Mathematics, 9th Edition, John Wiley \& Sons Inc.

Michael, N.; Zavlanos, M. M.; Kumar, V. \& Pappas, G. J. (2008). Distributed Multi-Robot task assignment and formation control, Proceedings of the IEEE International Conference on Robotics and Automation, pp. 128-133.

Michaud, F.; Letourneau, D.; Guilbert, M. \& Valin, J.-M. (2002). Dynamic robot formations using directional visual perception, Proceedings of the IEEE International Conference on Intelligent Robots and System, pp. 2740-2745.

Naffin, D. J.; Akar, M. \& Sukhatme, G. S. (2004). Lateral and Longitudinal Stability for Decentralized Formation Control, Proceedings of the International Symposium on Distributed Autonomous Robotic Systems, France.

Ogren, P. \& Leonard, N. E. (2003). Obstacle avoidance in formation, Proceedings of the IEEE International Conference on Robotics and Automation, Taipei, pp. 2492-2497.

Poduri, S. \& Sukhtame, G. S. (2007). Achieving Connectivity through Coalescence in Mobile Robot Networks, Proceedings of the first International Conference on Robot Communication and Coordination, Greece.

Processing website: http://www.processing.org

Sahin, E. \& Spears W. M. (2005). Swarm Robotics, Springer Lecture Notes in Computer Science 3342.

Seder, M. \& Petrovic, I. (2007). Dynamic Window based approach to mobile robot motion control in the presence of moving obstacles, Proceedings of the IEEE International Conference on Robotics and Automation, Italy, pp. 1986-1991.

Shao, J.; Wang, L. \& Xie, G. (2006). Flexible Formation Control for Obstacle Avoidance Based on Numerical Flow Field, Proceedings of the IEEE Conference on Decision and Control, pp. 5986-5991.

Traer Physics website: http://www.cs.princeton.edu/ traer/physics

Varghese, B. \& McKee, G. T. (2008). A Macroscopic Model for Multi-agent Pattern Formation, Proceedings of the 6th EuropeanWorkshop on Multi-agent Systems, Bath, UK, pp. 423-433.

Varghese, B. \& McKee, G. T. (2008). A Mathematical Model, Implementation and study of a swarm conglomerate and its formation control, Proceedings of the Towards Autonomous Robotic Systems Conference, Edinburgh, pp. 156-162. 
Wang, J.; Wu, X. - B. \& Xu, Z. - L. (2006). Decentralized formation control and obstacle avoidance based on potential field method, Proceedings of the fifth International Conference on machine Learning and Cybernetics, pp. 803-808. 


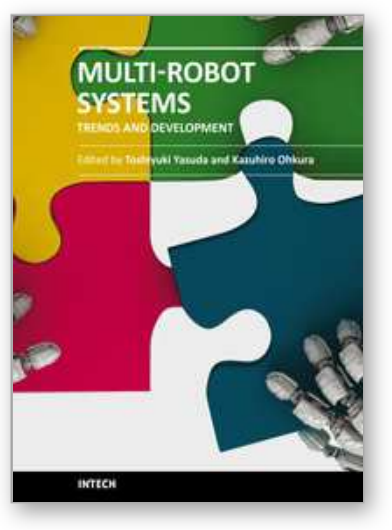

\author{
Multi-Robot Systems, Trends and Development \\ Edited by Dr Toshiyuki Yasuda
}

ISBN 978-953-307-425-2

Hard cover, 586 pages

Publisher InTech

Published online 30, January, 2011

Published in print edition January, 2011

This book is a collection of 29 excellent works and comprised of three sections: task oriented approach, bio inspired approach, and modeling/design. In the first section, applications on formation, localization/mapping, and planning are introduced. The second section is on behavior-based approach by means of artificial intelligence techniques. The last section includes research articles on development of architectures and control systems.

\title{
How to reference
}

In order to correctly reference this scholarly work, feel free to copy and paste the following:

Blesson Varghese and Gerard Mckee (2011). Swarm Patterns: Trends \& Transformation Tools, Multi-Robot Systems, Trends and Development, Dr Toshiyuki Yasuda (Ed.), ISBN: 978-953-307-425-2, InTech, Available from: http://www.intechopen.com/books/multi-robot-systems-trends-and-development/swarm-patterns-trendstransformation-tools

\section{INTECH}

open science | open minds

\section{InTech Europe}

University Campus STeP Ri

Slavka Krautzeka 83/A

51000 Rijeka, Croatia

Phone: +385 (51) 770447

Fax: +385 (51) 686166

www.intechopen.com

\section{InTech China}

Unit 405, Office Block, Hotel Equatorial Shanghai

No.65, Yan An Road (West), Shanghai, 200040, China

中国上海市延安西路65号上海国际贵都大饭店办公楼 405 单元

Phone: +86-21-62489820

Fax: $+86-21-62489821$ 
(C) 2011 The Author(s). Licensee IntechOpen. This chapter is distributed under the terms of the Creative Commons Attribution-NonCommercialShareAlike-3.0 License, which permits use, distribution and reproduction for non-commercial purposes, provided the original is properly cited and derivative works building on this content are distributed under the same license. 